\title{
Sling Effect in Collisions of Water Droplets in Turbulent Clouds
}

\author{
GREGORY FALKOVICH \\ Physics of Complex Systems, The Weizmann Institute of Science, Rehovot, Israel \\ Alain Pumir \\ Institut Non Lineaire de Nice, and Laboratoire J. A. Dieudonné, Université de Nice, and CNRS, Nice, France
}

(Manuscript received 12 December 2006, in final form 10 July 2007)

ABSTRACT

\begin{abstract}
The effect of turbulence on the collision rate between droplets in clouds is investigated. Because of their inertia, water droplets can be shot out of curved streamlines of the turbulent airflow. The contribution of such a "sling effect" in the collision rate of the same-size water droplets is described and evaluated. It is shown that already for turbulence with the dissipation rate $10^{3} \mathrm{~cm}^{2} \mathrm{~s}^{-3}$, the sling effect gives a contribution to the collision rate of $15-\mu \mathrm{m}$ droplets comparable to that due to the local velocity gradient. That may explain why the formulas based on the local velocity gradient consistently underestimate the turbulent collision rate, even with the account of preferential concentration.
\end{abstract}

\section{Introduction}

There is growing evidence that cloud turbulence increases the collision rate between water droplets, thus enhancing the creation and growth of raindrops. However, both qualitative understanding and quantitative treatment are far from satisfactory (see, e.g., Jonas 1996; Vaillancourt and Yau 2000; Shaw 2003; and references therein). In particular, the role of turbulence in inducing collisions of equal-size droplets is very important for the evolution of narrow droplet distribution that may be produced by condensation in cloud cores.

The collision rate $N\left(a, a^{\prime}\right)$ between the droplets or particles with the radii $a, a^{\prime}$ is the product of mean concentrations $n(a) n\left(a^{\prime}\right)$ times half the area of the collision sphere $2 \pi\left(a+a^{\prime}\right)^{2}$ times the modulus of the relative radial velocity of droplets before the contact $\Delta v$ and times the radial distribution function $P\left(a+a^{\prime}\right)$, which accounts for nonuniformities of the concentration field [i.e., mutual correlations between droplets (Sundaram and Collins 1997; Wang et al. 1998, 2005)]:

$$
N\left(a, a^{\prime}\right)=2 \pi\left(a+a^{\prime}\right)^{2} n(a) n\left(a^{\prime}\right) \Delta v P\left(a+a^{\prime}\right) .
$$

Corresponding author address: Alain Pumir, Laboratoire J. A. Dieudonné, Université de Nice, and CNRS, Parc Valrose, Nice 06108, France.

E-mail: alain.pumir@unice.fr
Since the aerosols vary in sizes from submicron to thousands of microns and cloud conditions vary widely (Grabowski and Vaillancourt 1999), collisions can be induced by different physical mechanisms. The relative velocity, $\Delta v$, can be due to Brownian motion, gravitational settling, and airflow gradients while the radial distribution $P$ is influenced by hydrodynamic interaction between approaching droplets and preferential concentration due to the airflow.

Here we focus on the contributions of the airflow to the collision rate. Specifically, we consider collisions of equal-size droplets that fall with the same velocity in a still air. ${ }^{1}$ We consider droplet sizes exceeding a few microns and neglect Brownian motion. For such droplets, the airflow is the sole source of relative velocity $\Delta v$ and it also influences $P(2 a)$ because of droplet inertia (called the effect of preferential concentration).

In spite of these simplifying assumptions, the problem remains very complicated since the collision rate depends on four dimensionless numbers. The first one is the ratio $\eta / a$, where $\eta$ is the viscous scale of turbulence. The other three parameters characterize air tur-

\footnotetext{
${ }^{1}$ There is a common misconception that the gravitational collision rate is zero for equal-size droplets. This is not so since hydrodynamic interaction changes settling velocities (e.g., a pair of close droplets falls faster). For a dilute set of droplets, such effects can be neglected though.
} 
bulence, droplet inertia, and gravity-Reynolds, Stokes, and Froude numbers, respectively:

$$
\operatorname{Re}=(L / \eta)^{4 / 3}, \quad \mathrm{St}=\tau \nu / \eta^{2}=\lambda \tau, \quad F=\lambda \eta / g \tau
$$

Here $L$ is the integral length scale (on the order of the size of the largest turbulent eddies, i.e., hundreds of meters or more), $\nu$ is the air viscosity, $g$ is the acceleration of gravity, $\lambda$ is the rms air-velocity gradient, and $\tau=(2 / 9)\left(\rho_{0} / \rho\right)\left(a^{2} / \nu\right)$ is called the response (Stokes) time with $\rho, \rho_{0}$ being the air and water density, respectively.

The simplest limit to describe is that of small droplets when $\mathrm{St} \ll 1$ and $F \gg 1$ (for the turbulence dissipation rate $\epsilon=\nu \lambda^{2} \simeq 10^{2} \div 10^{3} \mathrm{~cm}^{2} \mathrm{~s}^{-3}$, such a limit takes place for $a \leqq 5 \mu \mathrm{m})$. In this case, droplets move with the airflow. Therefore, they are distributed uniformly so that $P(2 a)=1$ while their relative velocity is determined locally by the air velocity, which is spatially smooth at such scales (since $a \ll \eta$ ) so that (Saffman and Turner 1956)

$$
\Delta v \sim \lambda a .
$$

Such a contribution to the collision rate is noticeable only for very energetic turbulence $\left(\epsilon>2 \times 10^{3} \mathrm{~cm}^{2} \mathrm{~s}^{-3}\right)$. However, it has been realized relatively recently that droplet inertia makes both $P$ and $\Delta v$ grow very fast with $\mathrm{St}$ in some interval of sizes. This can be attributed to the following two effects.

First, inertial particles cluster in random flows, which increases $P$. This effect of preferential concentration has been identified long ago and is still the subject of intensive study (see Maxey 1987; Squires and Eaton 1991; Wang and Maxey 1993; Sundaram and Collins 1997; Reade and Collins 2000; Kostinski and Shaw 2001; Jaczewski and Malinowski 2005; McFarquhar 2004; Franklin et al. 2005; Grits et al. 2006; and the references therein). Generally, the dimensionless quantity $P(2 a)$ depends in a complicated way on all four dimensionless parameters so the proper quantification of this effect and of its role in the collision rate enhancement remains to be done. However, for distances $l$ much less than $\eta$, it has been inferred from the data (Sundaram and Collins 1997) that $P(l)$ has a power-law dependence and has been argued theoretically (Balkovsky et al. 2001) that the dependence must be of the form $P(2 a) \sim(\eta / a)^{\alpha}$. The dimensionless quantity $\alpha$ depends on the three dimensionless parameters listed in (2). The behavior of $\alpha$ is well understood for small St where the fast growth $\alpha \simeq b(\mathrm{Re}, F) \mathrm{St}^{2}$ was predicted by Balkovsky et al. (2001), Falkovich et al. (2002), and confirmed by Falkovich and Pumir (2004), Chun and Koch (2005), and Chun et al. (2005) for moderate Re. Gravity is expected to reduce preferential concentration by diminishing the value of $\alpha$ (Falkovich et al.

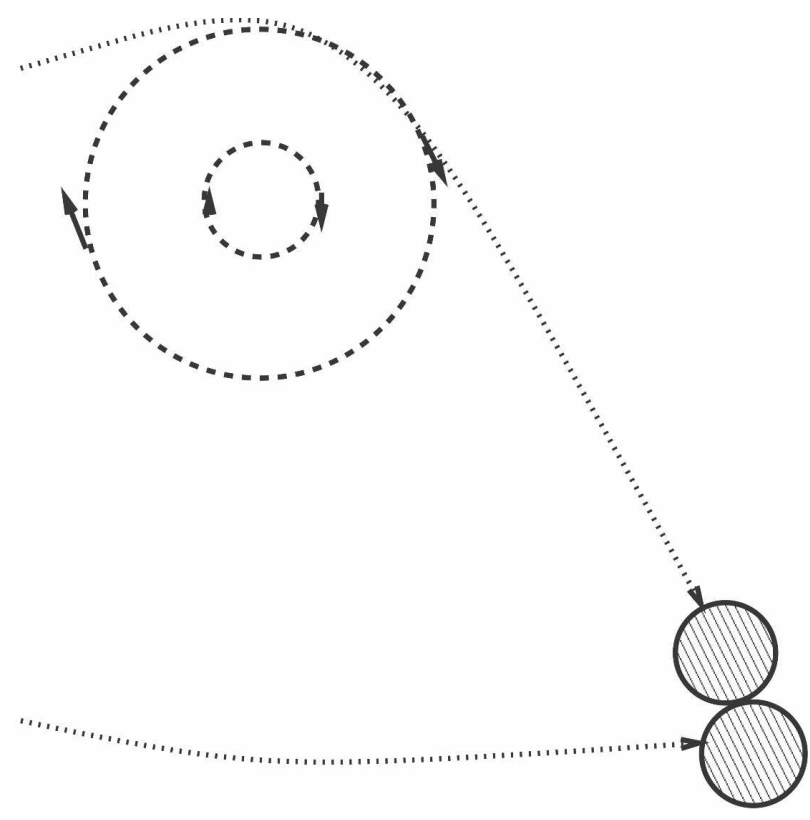

FIG. 1. Sketch of the sling effect. Dotted lines show the trajectories of the droplets while broken lines show the streamlines of the vortex in the airflow.

2002), an effect also confirmed numerically by Falkovich and Pumir (2004). In this context, our goal in this work is to establish $\alpha$ for larger St and Re than before and to find out at which droplet sizes the fast growth $\alpha(\mathrm{St})$ saturates.

Second, it has been predicted that already at small St there is a contribution to $\Delta v$ that does not depend linearly on the distance between droplets and is not determined by a local airflow gradient. Such contribution is expected to grow with $a$ much faster than linearly. Indeed, the Stokes number is the mean velocity gradient times the droplet response time. Even in a turbulent flow with a small St, there is a finite probability of velocity gradients comparable with $1 / \tau$. A droplet that encounters such a gradient can acquire a velocity that differs significantly from the air velocity and that of other droplets. Figure 1 illustrates this (so-called sling) effect: the right droplet passed through an intense vortex and had been thrown away as if by a sling. As a result, the relative velocity of the droplets at the point of collision may be determined not by the airflow gradient at this point [as in (3)] but rather by a distant vortex. As was first noticed by Falkovich et al. (2002), such events give an extra contribution to the collision rate not captured by the Saffman-Turner formula (even corrected for geometrical factors, inertia, and gravity by Wang et al. 1998, 2005, 2006; Dodin and Elperin 2002). The growth of this contribution with the Stokes number is expected to be of an activation form 
$\Delta v \propto \exp \left(-A / \mathrm{St}^{a}\right)$ [i.e., extremely fast (Falkovich et al. 2002; Wilkinson and Mehlig 2005; Derevyanko et al. 2006)]. Here we show that such activation dependence indeed takes place in realistic flows and that $a=1$. Let us give here some numbers adopted from the calculations to be described below. Consider turbulence with $\lambda=80 \mathrm{~s}^{-1}\left(\epsilon \simeq 960 \mathrm{~cm}^{2} \mathrm{~s}^{-3}\right)$. For droplets with $a=10$ $\mu \mathrm{m}, \mathrm{St}=0.08$ and sling effects are negligible; for $a=15$ $\mu \mathrm{m}, \mathrm{St}=0.2$ and the frequency of sling effects is $\simeq \lambda /$ 1000 while their contribution into the collision rate is about $20 \%$; for $a=20 \mu \mathrm{m}, \mathrm{St}=0.35$ and the frequency of sling effects is $\simeq \lambda / 50$ while their contribution into the collision rate is about $35 \%$ (the numbers correspond to Figs. 3, 5, 6 below). An independent confirmation that the Saffman-Turner formula (which disregards sling events) consistently underestimates the collision rate even at relatively small Stokes numbers has been obtained recently by direct numerical simulations (Franklin et al. 2005).

The main focus of this work is thus on the collision rate of the same-size droplets at small and moderate Stokes numbers. It corresponds, in particular, to the problem of rain initiation in warm clouds where $\epsilon=$ $10-10^{3} \mathrm{~cm}^{2} \mathrm{~s}^{-3}$ and the most interesting interval of sizes $a=5-25 \mu \mathrm{m}$ corresponds to the so-called condensation-collision bottleneck (growth of smaller droplets is typically due to condensation while for larger droplets gravitational collisions dominate already for $a^{\prime}-a>1$ $\mu \mathrm{m})$. Typical parameters of such clouds can be found in Grabowski and Vaillancourt (1999). While general analytic representation of the collision rate as a function of all four dimensionless variables does not seem to be feasible, the formulas $P(2 a) \sim(\eta / a)^{\alpha}$ and $\Delta v \sim$ $\exp (-A / \mathrm{St})$ do seem to capture the main dependencies in this region of parameters. Our specific focus is thus on two dimensionless quantities, $\alpha$ and $A$, which determine the fast growth of the collision rate with the Stokes number in this interval of droplet sizes. We also wish to quantify the role of gravity, which is expected to weaken the interaction between droplets and airflow and to suppress the effects studied here.

Note in passing that a generalization for the different-size droplets value, $P\left(a, a^{\prime}\right)$, has been suggested in Falkovich et al. (2002), Bec et al. (2005), and Chun et al. (2005), who all conclude that the power law saturates for small separations. For different-size droplets, inertia also leads to an extra contribution into the relative velocity [considered at small St by Saffman and Turner (1956) and not treated here].

\section{Modeling approach and numerical methods}

The most straightforward way to model the collision rate is with the discrete finite-size droplets embedded into a properly modeled flow (see, e.g., Franklin et al. 2005; Wang et al. 2005). Resolution requirements restrict the possibility of doing direct numerical simulation (DNS) to limited values of the Reynolds numbers. Also, because the concentration of droplets is quite low, obtaining reliable collision rates requires very long integration times. Moreover, computations with discrete droplets do not allow one to distinguish between local contributions to collisions (which must be described by the Saffman-Turner formula with a proper preferential concentration correction) and a sling effect contribution. These constraints impede progress toward a proper parameterization of the collision rate.

\section{a. Formulation of the problem}

Here we propose another complementary way of modeling based on the continuous description of the flow of droplets. The equation for the droplet velocity $\mathbf{v}$,

$$
d \mathbf{v} / d t=(\mathbf{u}-\mathbf{v}) / \boldsymbol{\tau}+\mathbf{g},
$$

can be considered to define everywhere in space the field $\mathbf{v}(\mathbf{r})$ from the known airflow field $\mathbf{u}(\mathbf{r})$.

In this problem, the airflow is incompressible and smooth; that is, all components of the matrix of the air velocity derivatives, $s_{i j}=\partial u_{i} / \partial r_{j}$, are finite and $\Sigma s_{i i}=0$. On the contrary, the flow of droplets is generally compressible and contains regions of infinite gradients (caustics produced by the sling effect). In the continuous description we are able to see clearly the sling events, which appear in the equations of motion for droplets as the crossing of trajectories (so the velocity field becomes multivalued) or as finite-time singularities of the continuous equations. Differentiating (4), one gets the equation for the matrix of droplet-flow gradients, $\sigma_{i j}=\partial v_{i} / \partial r_{j}$, in the comoving (Lagrangian) reference frame:

$$
\dot{\sigma}_{i j}+\sigma_{i k} \sigma_{k j}+\sigma_{i j} / \tau=s_{i j} / \tau .
$$

Nonlinearity (which corresponds to inertia) in the equation for $\hat{\sigma}$ leads to the possibility of divergence in a finite time of $\sigma$, where some component of $\sigma_{i j}$ turns into $-\infty$ in a finite time according to $\sigma \propto\left(t-t_{0}\right)^{-1}$ (Falkovich et al. 2002).

Because of spatial and temporal nonlocality, the analytical description of the sling effect contribution into the collision rate is difficult and has been done only for the simplest models. Assuming the airflow gradients are long correlated (i.e., the correlation time of $s$ exceeding $1 / s$ ) and solving (5) for constant $s$, one concludes that the probability of having $\sigma<-1 / \tau$ is equal to the probability of having $s<-1 / \tau$. For Gaussian statistics of $s, P(s) \propto \exp \left[-(s / 2 \lambda)^{2}\right]$, the probability of 
explosion behaves as $\exp \left(-1 / \mathrm{St}^{2}\right)$ (Falkovich et al. 2002). In the opposite limit of a short-correlated $s$, the probability of such events is found to be $\exp (-A / \mathrm{St})$ (Wilkinson and Mehlig 2003, 2005; Derevyanko et al. 2006). Since real turbulent flow is neither short nor long correlated, our first task is to find how exactly the probability of explosions and their contribution to the collision rate grows with $\mathrm{St}$ in real flows. We show below that it behaves as $\exp [-A(\mathrm{Re}, F) / \mathrm{St}]$. Particularly important is understanding the dependence of $A$ on the Reynolds number, which varies by many orders of magnitude in atmospheric flows. In this paper, we perform direct numerical simulations of the airflow turbulence at Reynolds numbers $\operatorname{Re}_{\lambda}$ that were never reached before in collision rate calculations. We pay the price by not performing kinetic droplet simulations (i.e., following separate droplets), using instead a continuous description based on (4) and (5) and the equation for droplet concentration in the Lagrangian reference frame,

$$
d n / d t=-n \sigma_{i i}(t) .
$$

The continuous description is meaningful only at the scales exceeding droplet sizes. The properties of the coarse-grained distribution at a given scale $r$ can be estimated by using the method developed by Balkovsky et al. (2001), Falkovich et al. (2002), and implemented numerically by Falkovich and Pumir (2004) in the restricted case where the Stokes number was small and where no sling contribution was expected. That method requires one to follow, in addition to $\mathbf{x}, \mathbf{v}, \boldsymbol{\sigma}$, and $n$, the deformation tensor $\mathbf{W}(t)$ induced by the velocity field $\mathbf{v}$. Consider the tensor $\mathbf{W}(t)$ that describes how a small line element, $\delta \mathbf{I}(|\delta \mathbf{I}| \ll \eta)$ is transported by the flow: $\delta \mathbf{I}(t)=$ $\mathbf{W}(t) \cdot \delta \mathbf{I}(0)$. The inverse of the tensor $\mathbf{W}, \mathbf{W}^{-1}$ therefore maps the line element $\delta \mathbf{I}(t)$ at time $t$ to its origin at time $t=0$. The growth of $\mathbf{W}^{-1}$ is thus associated with the contraction of the line element $\delta \mathbf{I}(t)$ while $\operatorname{det}(\mathbf{W})$ determines the contraction rate of the volume along droplet trajectories. The evolution equation for $\mathbf{W}^{-1}$ is derived from the evolution equation for $\delta \mathbf{I}(t): d \delta \mathbf{I}_{i} / d t=$ $\partial v_{i} / \partial x_{j} \cdot \delta \mathbf{l}_{j}(t):$

$$
\frac{d \mathbf{W}^{-1}}{d t}=-\mathbf{W}^{-1} \cdot \boldsymbol{\sigma}
$$

To estimate the contribution of a trajectory to the coarse-grained droplet density at scale $r$, the integration is carried out until $\left|\mathbf{W}^{-1}\right|$ reaches $(\eta / r)$, where the norm considered here is the usual Euclidean norm. At this point, the value of $n$ coarse-grained at scale $r$ is recorded. The density $n$ at scale $h$ is taken to be equal to the mean concentration; that is, we neglect (rather small) intermittency corrections observed in the inertial range (Bec et al. 2007). The Eulerian value of the $k$ th moment is obtained by averaging the values of $n^{k-1}$ over all the trajectories computed. In our calculation, the Eulerian quantities are estimated when the value of $\left|\mathbf{W}^{-1}\right|$ along a trajectory has grown by a factor $\eta / a$. The quantities to be determined in this way (the parameter $\alpha$ and the Lyapunov exponent of the droplet flow) depend on the logarithm of that factor so order-unity indeterminacy in its definition is unimportant.

The occurrence of a sling effect results in a singularity of $\boldsymbol{\sigma}$ in a finite time, $\boldsymbol{\sigma} \propto\left(t-t_{0}\right)^{-1}$, which in turn leads to a divergence of the droplet density $[n \propto(t-$ $\left.t_{0}\right)^{-1}$; see Eq. (6)]. Physically, neither the droplet velocity gradient nor the droplet density can grow unbounded since droplets have a finite size, $a$, and they cannot come arbitrarily close to one another. Therefore, the droplet velocity gradients do not grow more than by a factor $\sim(\eta / a)$. Once the gradient has reached this predetermined threshold value, the equation is regularized by flipping the sign of $\boldsymbol{\sigma}$. This simply corresponds to a fast droplet passing a slow one so that their velocity difference (as well as the gradient) changes sign. Similarly, the density increases until the time of the flip and then decreases. This algorithm leads to a numerically well-posed problem.

In the approach developed here, the collision rate, resulting from the motion of droplets, evolving according to Eq. (4), is modeled by separating explicitly the "continuous" contributions from the "sling" events. Such separation is meaningful only at not very large Stokes numbers when sling events are rare. The "continuous contribution" is proportional to the local velocity gradient $\boldsymbol{\sigma}$ (as in the Saffman-Turner approach). Given the value of $n$ and of $\sigma$ at a given time, the instantaneous flux of incoming droplets toward a given droplet is as follows:

$$
\Phi_{\text {cont }}(t)=(2 a)^{3} n(t) \int_{\hat{\mathbf{e}} \cdot \boldsymbol{\sigma} \cdot \hat{\mathbf{e}}<0}(\hat{\mathbf{e}} \cdot \boldsymbol{\sigma} \cdot \hat{\mathbf{e}}) d \Omega,
$$

where $\hat{\mathbf{e}}$ is the unit normal vector to the sphere and $d \Omega$ is the solid angle that measures the area on the sphere. The contribution to the collision rate, $K_{\text {cont }}$, along a trajectory is simply obtained by averaging $\Phi_{\text {cont }}$ over many trajectories and over time. Because the growth of $n$ is accounted for in the resulting contribution for the collision term, the influence of the sling effect on concentration (because of caustics left after sling events) is partially taken into account in this formula. However, as has been pointed out above, the sling effect also results in an additional contribution to the velocity difference, which is not proportional to the local velocity 
gradient. As suggested by Fig. 1, a sling event involves a number of droplets incoming in a small region with significantly different velocities, a situation leading to an outbursts of collisions, which we estimate as follows. The source term in Eq. (5), necessary to start a blow-up process, $s / \tau$, should be large enough: $|s|>1 / \tau$. Based on the Kolmogorov picture of fully developed turbulence, the extent of the region where gradients reach the value $s$ on the order or larger of $1 / \tau$ is on the order of $l \sim$ $(\nu \tau)^{1 / 2}=\eta \mathrm{St}^{1 / 2}$. The time over which the collision takes place is estimated to be on the order of the droplet relaxation time, $\tau$. Last, the range of droplet velocities involved during the collision can be estimated as $|\delta \mathbf{v}| \sim$ $l / \tau$. Based on these estimates, the number of collisions that occur in the wake of a sling event that have happened at time $t_{s}$ can be estimated as follows:

$$
N_{\text {sling }}(t)=4 \pi(2 a)^{2} \times n\left(t_{s}+\tau / 2\right) \times|\delta \mathbf{v}| \times \tau .
$$

The relaxation time of droplet velocities is $\tau$ and this is a typical duration of the time when droplets have substantially different velocities and sling effect contribution appears. That is why in estimating $N_{\text {sling, }}$, the density of droplets is taken at a time $\tau / 2$ after the sling event time $t_{s}$, which provides a reasonable estimate for the droplet density during the entire process. As is clear from the heuristic derivation above, the value of $N_{\text {sling }}$ obtained in such a way is up to a numerical factor of order unity, whose precise value could be estimated by kinetic numerical simulations, which is beyond the scope of this work. In practice, the number of collisions due to sling effects, divided by $\left(32 \pi a^{3}\right)$, is computed by adding after each blow up along the droplets' trajectories, at time $t_{s}$, a term $\propto n\left(t_{s}+\tau / 2\right) \mathrm{St}^{1 / 2}(\eta / 2 a)$. The ratio $\mathrm{St}^{1 / 2}(\eta / 2 a)$ is a number of the order 10 for the range of physical situations we are interested in. The collision rate is obtained by dividing the result by the time over which the droplet is tracked. In the rest of this paper, we estimate separately the contributions from the continuous term and the sling contribution, given by Eqs. (8) and (9), respectively.

\section{b. Numerical methods}

To this end, we generate a statistically stationary turbulent flow in a cube with periodic boundary conditions. The equations are solved with a pseudo-spectral code (see Pumir 1994 for details). All the appropriate length scales of the flow are adequately resolved. We work in the range of microscale Taylor-Reynolds number, $R_{\lambda}$ (Pumir 1994) $21 \leq R_{\lambda} \leq 105$. In this turbulent flow, we follow the motion of inertial droplets by solving the equation for the position, $d \mathbf{x} / d t=\mathbf{v}$, along with (4). We use the initial condition $\mathbf{v}=\mathbf{u}$. The Eq. (5) for the tensor of droplet velocity derivatives is integrated along the way. The integration of Eqs. (4) and (5) requires the interpolation of the fluid velocity, $\mathbf{u}$, or its derivative, $s$, from the numerical mesh to the droplet position. This is done by using spline interpolation techniques (see Girimaji and Pope 1990). The equations are solved by using algorithms that are second-order accurate in time, or higher.

The equations of motion were integrated for several Stokes numbers (ranging from $\mathrm{St}=0.05$ to $\mathrm{St} \approx 5$ ), and the effect of gravity was explicitly taken into account. Warm clouds correspond to low latitudes so the acceleration of gravity is constant $\left(g \approx 9.8 \mathrm{~m} \mathrm{~s}^{-2}\right)$, while the intensity of turbulence depends on the type of cloud (Grabowski and Vaillancourt 1999; see also Franklin et al. 2005). One can characterize the relative importance of gravity versus turbulence by the parameter $\epsilon_{0} \equiv$ St $\times$ $F$, which is the ratio of respective accelerations. Note that $\epsilon_{0}$ does not depend on the characteristics of the droplet. In our modeling we made two sets of runs corresponding to two levels of turbulence, one corresponding to $\epsilon \simeq 600 \mathrm{~cm}^{2} \mathrm{~s}^{-3}, \epsilon_{0}=0.2$ and $\epsilon \simeq 1500$ $\mathrm{cm}^{2} \mathrm{~s}^{-3}, \epsilon_{0}=0.4$. As done in Falkovich and Pumir (2004), we consider droplets in a fixed environment by fixing the value of $\epsilon_{0}$ and the Reynolds number. We study the dependencies of different quantities on the size of the droplet parameterized by St.

\section{Numerical results}

The method sketched above to estimate the coarsegrained properties at a scale $r$ is then used to evaluate the collision rate as a function of scale. More precisely, consider the droplets of radius $a$. To compute the collision rate, the continuous and sling contributions to the collision rate are computed along trajectories, until the compression, given by $\left|\mathbf{W}^{-1}\right|$ [see Eq. (7)], reaches the value $\eta / a$. The various contributions coming from different trajectories are then accumulated, and the mean value of the collision rate is extracted.

The way the flow leads to the compression of an ensemble of droplets, described by Eq. (7), plays a crucial importance in the physical processes controlling droplet collision rates. The numerical results indicate that the averaged value of $\ln \left(\left|\mathbf{W}^{-1}\right|\right)$ grows linearly with time, with the growth rate $\lambda_{3}$, defined by $\left\langle\ln \left(\left|\mathbf{W}^{-1}\right|\right)\right\rangle=$ $-\lambda_{3} t$, where the angle brackets denote an averaged quantity. The growth rate gives direct access to the most negative Lyapunov exponent $\lambda_{3}$ that governs the strongest contraction in the flow. At a given Reynolds number, the value of $-\lambda_{3} \times \tau_{K}$ has a nontrivial dependence on the Stokes number. It starts at a value $-\lambda_{3} \times$ $\tau_{K} \approx 0.16$ at St $=0$ (Falkovich and Pumir 2004), then increases up to $\mathrm{St} \sim 0.5$, leading to a twofold increase of 


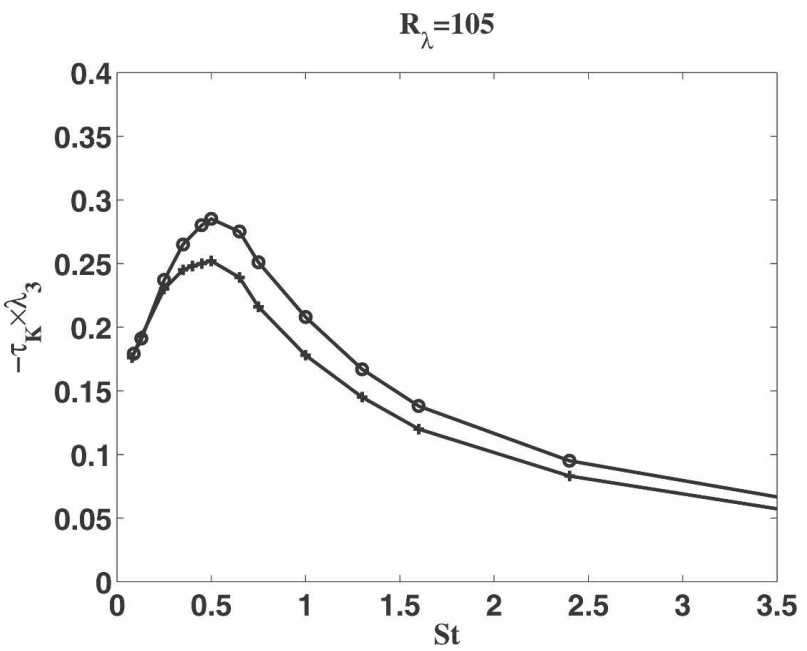

FIG. 2. The contraction rate along trajectories of particles at $R_{\lambda}=105$ and $\epsilon_{0}=0.4$ ( $\bigcirc$ symbols $)$ and $\epsilon_{0}=0.2$ ( + symbols $)$.

$\lambda_{3}$, compared to its value when $\mathrm{St}=0$, before decreasing at higher values of St (see Fig. 2). Qualitatively similar dependence has been found for a model shortcorrelated flow (Wilkinson and Mehlig 2003). The values of $\lambda_{3}$ are not affected much by the gravity in the range considered in this work $\left(\epsilon_{0} \geq 0.2\right)$; the results for $\epsilon_{0}=5$ (not shown) are within $10 \%$ from those shown in Fig. 2 for $\epsilon_{0}=0.4$.

Sling events, in our approach, are manifested by divergences of the droplet velocity derivative tensor, $\boldsymbol{\sigma}$. The blow-up frequency, $f_{\mathrm{bu}}$, defined as the total number of sling events divided by the integration time, plays here a crucial role. Figure 3 shows $f_{\text {bu }}$ multiplied by the Kolmogorov time $\tau_{K}$. The blow-up occurrences are induced by large velocity gradients, of typical size $(\epsilon / \nu)^{1 / 2}=\tau_{\kappa}^{-1}$. This suggests that the product $f_{\mathrm{bu}} \times \tau_{K}$ should depend mostly on St and $\epsilon$ but relatively little on the Reynolds number. This expectation is confirmed by our numerical results (see Fig. 3). No blow up is observed at very low values of St (for St $\lesssim 0.15$ ). As St increases, the value of $f_{\text {bu }}$ raises to a maximum value for $\mathrm{St} \sim 1.5$, then decreases slowly. The recent works on simple versions of the problem (one-dimensional and short-correlated flows) suggest the dependence of the blow-up frequency as a function of St on the form $f_{\text {bu }} \approx$ $\exp (-A / \mathrm{St}$ ) (Wilkinson and Mehlig 2005; Derevyanko et al. 2006). Here we find empirically that the curve could be fit pretty well by the dependence of the form:

$$
f_{\mathrm{bu}} \times \tau_{\kappa}=\mathrm{St}^{-2} \times \exp (-A / \mathrm{St}) \times\left(B+C \mathrm{St}^{c}\right) .
$$

The coefficient $A$ is found to decrease slightly as the Reynolds number increases $\left(A=2.1\right.$ for $R_{\lambda}=45, A=$ 1.85 for $R_{\lambda}=83$, and $A=1.70$ for $R_{\lambda}=105$ ), consistent

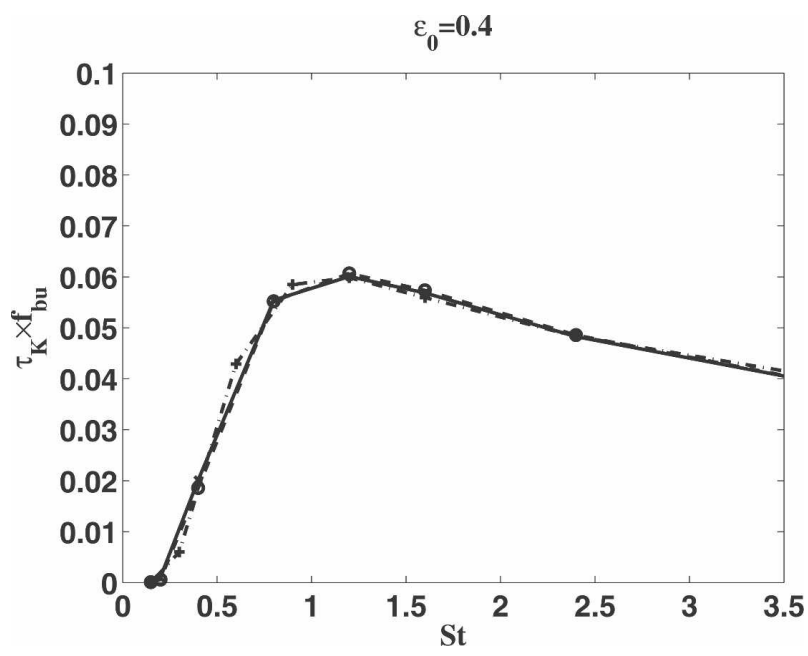

FIG. 3. The blow-up frequency as a function of the Stokes numbers for several Reynolds numbers: $R_{\lambda}=45$ (dotted-dashed line, + symbols), 83 (dashed line, $\bigcirc$ symbols), and 105 (full line, $\times$ symbols). The gravity is such that $\epsilon_{0}=0.4$.

with the fact that as turbulence becomes more intense, higher gradients appear in the flow, which are able to induce the blow up of $\boldsymbol{\sigma}$ at increasingly low values of St. Yet, at higher values of the Stokes number, the blow-up frequency seems to decrease as the value of the Reynolds number increases, a somewhat surprising effect. Upon increasing gravity (decreasing $\epsilon_{0}$ ), the blow-up frequency generally goes down, with a similar Stokes number dependence. This trend can be understood qualitatively by noticing that as gravity increases, the source term on the right-hand side of Eq. (5) becomes decorrelated, resulting in a diminished blow-up frequency.

The dependence of the coarse-grained droplet density, $\left\langle n^{2}\right\rangle_{r}$, as a function of $\eta / r$ is very similar to the one obtained by Falkovich and Pumir (2004). Namely, $\left\langle n^{2}\right\rangle_{r}$ has essentially a power-law dependence as a function of $\eta / r$. The exponent $\alpha$ of the exponent is plotted here as a function of St at $R_{\lambda}=83$, and for the values of $\epsilon_{0}=$ 0.2 and $\epsilon_{0}=0.4$. The value of the exponent increases sharply as a function of St up to St $\approx 1$, where it starts to saturate and decrease slightly. The qualitative aspect of the dependence of $\alpha$ as a function of St does not depend on the precise value of the Reynolds number in the range of $R_{\lambda}$ studied. We find that at values of $\mathrm{St} \geq$ 0.1 , the value of the exponent is somewhat higher than the one found by Falkovich et al. (2002; see Fig. 4). This difference can be attributed to the fact that the exponent there was computed by studying contraction along the fluid trajectory, which in the limit St $\rightarrow 0$, differs very little from the droplets trajectories. Quantitative differences remain even for values of $\mathrm{St}$ as small as $\mathrm{St} \approx$ 


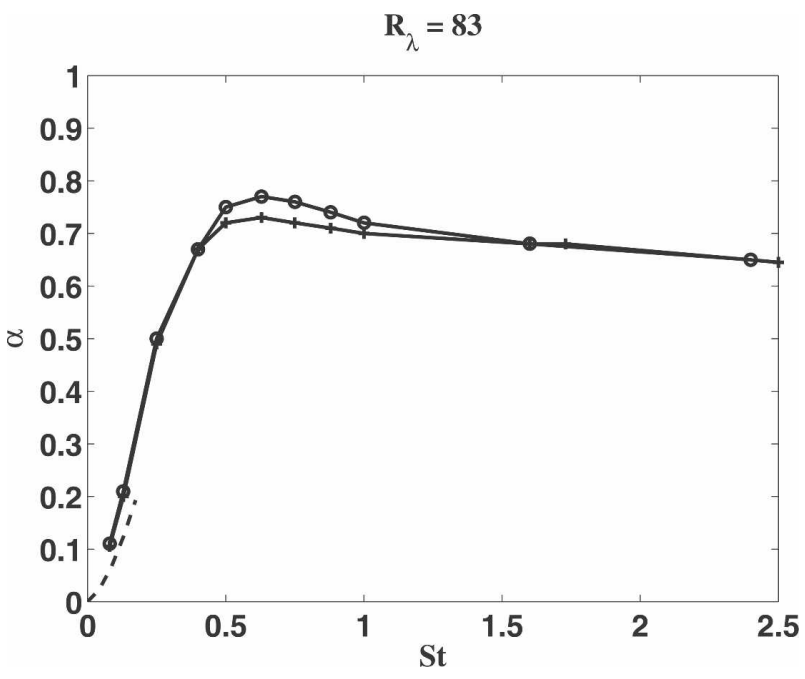

FIG. 4. The St dependence of the exponents $\alpha$ obtained by fitting $\left\langle n^{2}\right\rangle_{r}$ by a power-law dependence as a function of $r:\left\langle n^{2}\right\rangle_{r} \propto$ $(\eta / r)^{\alpha}$, at $\epsilon_{0}=0.4$ (upper curve, $\bigcirc$ symbols) and $\epsilon_{0}=0.2$ (lower curve, + symbols). The value of the exponent obtained by Falkovich and Pumir (2004) at small values of St is shown by the dashed line. The error bars on the data points are less that $5 \%$.

0.1. At moderate values of St, the exponent is larger when gravity is weaker relative to turbulence (larger $\epsilon_{0}$ ), as expected (Falkovich et al. 2002).

Finally, Fig. 5 shows the continuous contribution to the collision rates, normalized by $32 \pi a^{3} / \tau_{K}$, as a function of the Stokes numbers. The Saffman-Turner (ST) formula [which by itself is an upper bound according to Andersson et al. (2007)] gives in our units $\tau_{\kappa} \times K_{\mathrm{cont}}^{\mathrm{ST}} t$ $\left(32 \pi a^{3}\right)=(1 / 30 \pi)^{1 / 2}$, the value indicated by the horizontal dashed line. The continuous contribution starts at $\mathrm{St} \rightarrow 0$, very close to this value, and increases to a maximum value at $S t \approx 1$ before decreasing very slowly. Increasing the relative role of gravity (decreasing $\epsilon_{0}$ ) tends to decrease the collision rate. Over the range of parameters studied here, it was found that the continuous part of the collision rate increases when the Reynolds number increases. Our runs at different values of $R_{\lambda}$ all suggest that the values of $K_{\text {cont }}$ are always larger than predicted by the Saffman-Turner formula, for St $>0$ (i.e., when inertia plays a role).

The sling contribution to the collision rate, shown in Fig. 6 at the value of the Reynolds number $R_{\lambda}=105$, starts from essentially zero at very small values of the Stokes number (the probability of having a sling effect is practically zero at $\mathrm{St} \ll 1$ ). Again, similar to what has been observed for the continuous contribution to the collision term, the sling contribution increases to a maximum at $\mathrm{St} \sim 0.8$. The phenomenological description of the sling collision rate used in this work is not expected to hold at values of the Stokes numbers larger
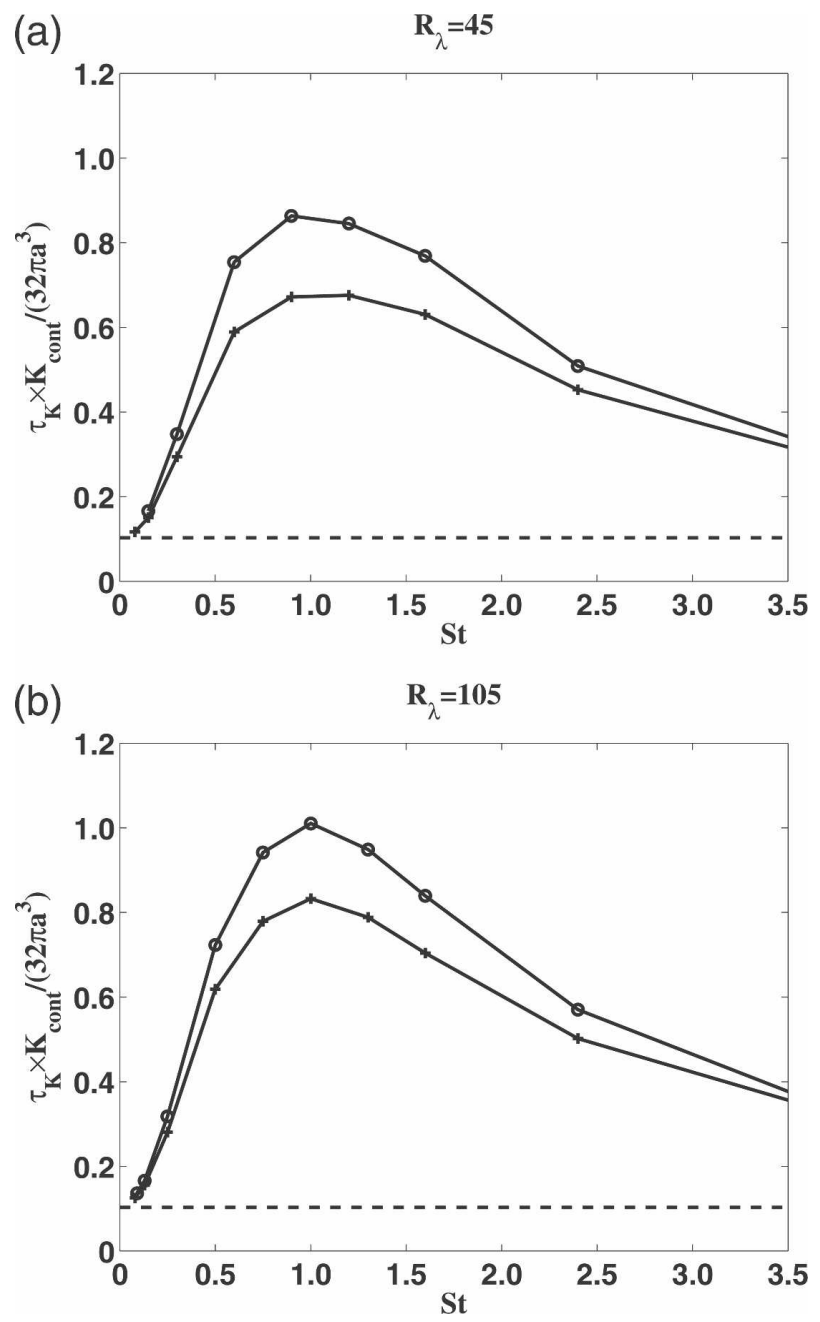

FIG. 5. The St dependence of the continuous component of the collision rate, normalized by the particle size $a$ and by the Kolmogorov time scale, $\tau_{K}$, at two values of gravity for Reynolds numbers of (a) $R_{\lambda}=45$ and (b) $R_{\lambda}=105: \epsilon_{0}=0.4$ (upper curve, the data points are plotted with $\bigcirc$ symbols) and $\epsilon_{0}=0.2$ (lower curves, the data points are plotted with + symbols). The horizontal dashed line corresponds to the Saffman-Turner formula. The collision rate peaks at a value $\mathrm{St} \approx 1$. The errors on the points are less than $5 \%$.

than $\sim 1$. In fact, our approach is based on the implicit assumption that droplets can be described by an essentially smooth hydrodynamic representation. This assumption becomes questionable as soon as $\mathrm{St} \gtrsim 1$. For this reason, only the part of the curve corresponding to values of $\mathrm{St} \leq 1.0$ has been shown. Figure 5 is meant to show the main trend, at moderate Stokes numbers [the formula used to define this term, Eq. (9), is defined up to a constant]. These data do not allow us to test the simple approximation for the sling contribution suggested by Wilkinson et al. (2006), $\mathrm{St}^{-1 / 2} \exp (-A / \mathrm{St})$, which fits the data for a synthetic velocity field without 


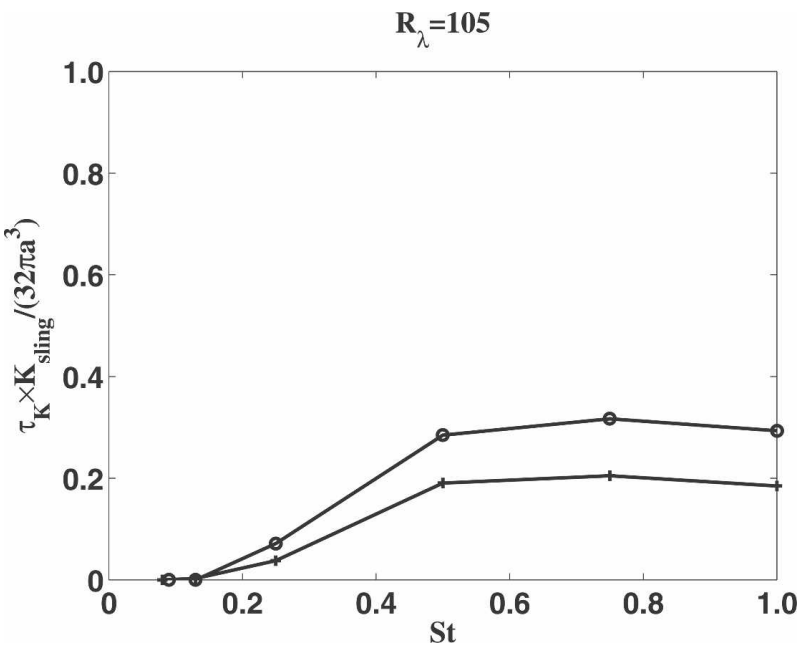

FIG. 6. The St dependence of the sling component of the collision rate, normalized by the particle size $a$ and by the Kolmogorov time, $\tau_{K}$, at two values of gravity: $\epsilon_{0}=0.4$ (upper curve, the data points are plotted with $\bigcirc$ symbols) and $\epsilon_{0}=0.2$ (lower curves, the data points are plotted with + symbols), at a Reynolds number of $R_{\lambda}=105$. The errors are on the order of $10 \%-15 \%$.

gravity well. Last, the limited range of the Reynolds number available numerically leaves open the question of the asymptotic limit of the collision rate as the Reynolds number tends to infinity.

Our method to estimate the collision rates, although based on procedures that can be formally justified for small Stokes number droplets, is very indirect. It is therefore appropriate to compare our estimates for the collision rates with the numerical results obtained by other groups. Franklin et al. (2005) and Wang et al. (2005), by using direct numerical simulations, estimated directly the collision rate among droplets. We ran a simulation at a value of $R_{\lambda}=45$ and $\epsilon_{0}=0.2$ (run Fa) comparable to run 3 of Franklin et al. at $R_{\lambda}=48$ and $\epsilon_{0}=0.21$, and simulations at $R_{\lambda}=40$ and $\epsilon_{0}=0.142$ (run Wa). The geometric collision rate is the rate of collision per droplet in the flow; it can thus be compared directly with the collision rates computed by the method of the present work, once the dimensionless values of $\Gamma$ obtained with our method have been made dimensional with the proper values of $\tau_{K}$ and $a$. The results are shown in Table 1. At the lowest value of $\mathrm{St}$ for run F, no sling contribution is expected, as indicated in the second line of Table 1 . The values of $\Gamma$ and $K_{\text {cont }}$ coincide to within $\sim 10 \%$. The most likely source of differences between the predictions of our method and the results obtained by Franklin et al. (2005) and Wang et al. (2005) can be attributed to the fact that we systematically neglected here intermittency corrections in the inertial range, thus underestimating slightly the collision rates. This could be in principle easily fixed. For run $\mathrm{F}$, at the highest value of the Stokes number, one finds that the continuous part of the collision rate, $K_{\text {cont }}$, underestimates the value found in Franklin et al. On the other hand, a significant sling effect is expected at this value of the Stokes number. Our method provides only an order of magnitude estimate of the sling term, that is, an estimate up to a numerical factor of order unity. The comparison of the prediction of our method with the data corresponding to run $\mathrm{W}$ also demonstrates that the sling term leads to a very significant contribution to the collision rate. The systematic comparison of the results obtained by directly counting the collision rates with the predictions of our method suggests that our parameterization provides a correct order of magnitude estimate. For more precision, it needs to be refined (possibly by an empirical orderunity factor).

\section{Conclusions}

In conclusion, we have studied the collision rates induced by turbulent air motion, considering how a homogeneous, isotropic turbulent flow induces collisions of inertial droplets. The method used in this work is essentially Lagrangian. We follow droplets advected in the flow, compute directly the flux of incoming droplets (continuous contribution), and estimate the number of collisions that occur in the aftermath of a "sling" effect.

TABLE 1. Comparison between the numerical estimates of collision rates by Franklin et al. (2005, their Table 5, run 3, denoted "F" here) and Wang et al. (2005, data from their Table 3, denoted "W") and the present estimates (runs Fa and Wa).

\begin{tabular}{|c|c|c|c|c|c|c|c|}
\hline Run & $R_{\lambda}$ & $a$ & St & $\epsilon_{0}$ & $\Gamma\left(\mathrm{cm}^{3} \mathrm{~s}^{-1}\right)$ & $\begin{array}{c}K_{\text {cont }} \\
\left(\mathrm{cm}^{3} \mathrm{~s}^{-1}\right)\end{array}$ & $K_{\text {sling }}\left(\mathrm{cm}^{3} \mathrm{~s}^{-1}\right)$ \\
\hline $\mathrm{F}$ & 48 & $10 \mu \mathrm{m}$ & 0.08 & 0.21 & $1.0 \times 10^{-6}$ & & \\
\hline $\mathrm{Fa}$ & 45 & & 0.08 & 0.2 & 0 & $0.85 \times 10^{-6}$ & 0 \\
\hline $\mathrm{F}$ & 48 & $20 \mu \mathrm{m}$ & 0.32 & 0.21 & $59.0 \times 10^{-6}$ & & \\
\hline $\mathrm{Fa}$ & 45 & & 0.30 & 0.2 & & $22.0 \times 10^{-6}$ & $10.0 \times 1.10^{-6}$ \\
\hline $\mathrm{W}$ & 40 & $20 \mu \mathrm{m}$ & 0.254 & 0.142 & $22.2 \times 10^{-6}$ & & \\
\hline Wa & 40 & & 0.254 & 0.14 & & $10.0 \times 10^{-6}$ & $1 \times 1.10^{-6}$ \\
\hline $\mathrm{W}$ & 40 & $25 \mu \mathrm{m}$ & 0.396 & 0.142 & $99.5 \times 10^{-5}$ & & \\
\hline Wa & 40 & & 0.396 & 0.14 & & $33.0 \times 10^{-6}$ & $11.0 \times 10^{-6}$ \\
\hline
\end{tabular}


The ratio of the collision rate to the Saffman-Turner formula is found to increase significantly from 1 to $\sim 10$ when the Stokes number increases from $\mathrm{St} \approx 0$ to $\mathrm{St} \approx$ 1 . The increase becomes more pronounced as the Reynolds number becomes larger.

In the range of Reynolds numbers studied here, sling contributions are negligible at very small Stokes numbers: their probability goes as $\exp (-A / \mathrm{St})$ as a function of St, with a coefficient $A$ of the order 1 . In practice, they become significant for Stokes numbers St $\gtrsim 0.20$.

The actual collision rates computed in this work are consistent, at $R_{\lambda} \approx 45$, with the recent results obtained by Franklin et al. (2005). In particular, our results allow us to disentangle the contributions due to the sling events, which we find to be quite significant for droplets of size $a=20 \mu \mathrm{m}$.

This work should help to clarify the origin of the enhancement of the collision rates of inertial droplets due to turbulence, and also ultimately, to devise a parameterization of this collision rate.

Acknowledgments. We acknowledge support from the Israel Science Foundations and from the European Commission (Contract HPRN-CT-2002-00300) and from IDRIS for computer time. G. Falkovich is grateful to A. Kostinski and A. Pumir to S. Malinowski for useful discussions. The authors are also very grateful to the reviewers for constructive and generally very useful comments.

\section{REFERENCES}

Andersson, B., K. Gustavsson, B. Mehlig, and M. Wilkinson, cited 2007: Advective collisions. [Available online at http://lanl. arxiv.org/abs/nlin/0702024.]

Balkovsky, E., G. Falkovich, and A. Fouxon, 2001: Intermittent distribution of inertial particles in turbulent flows. Phys. Rev. Lett., 86, 2790-2793.

Bec, J., A. Celani, M. Cencini, and S. Musacchio, 2005: Clustering and collisions of heavy particles in random smooth flows. Phys. Fluids, 17, 1-11.

—, L. Biferale, M. Cencini, A. Lanotte, S. Musacchio, and F. Toschi, 2007: Heavy particle concentration in turbulence at dissipative and inertial scales. Phys. Rev. Lett., 98, 1-4.

Chun, J., and D. L. Koch, 2005: Coagulation of monodisperse aerosol particles by isotropic turbulence. Phys. Fluids, 17, $1-15$.

,-- S. L. Rani, A. Ahluwalia, and L. Collins, 2005: Clustering of aerosols particles in isotropic turbulence. J. Fluid Mech., 536, 219-251.

Derevyanko, S., G. Falkovich, K. Turitsyn, and S. Turitsyn, 2006: Explosive growth of inhomogeneities in the distribution of droplets in a turbulent air. J. Turb., 8, 1-18.

Dodin, Z., and T. Elperin, 2002: On the collision rate of particles in turbulent flow with gravity. Phys. Fluids, 14, 2921-2924.

Falkovich, G., and A. Pumir, 2004: Intermittent distribution of heavy particles in a turbulent flow. Phys. Fluids, 16, L47-L50.
_ A. Fouxon, and M. G. Stepanov, 2002: Acceleration of rain initiation by cloud turbulence. Nature, 419, 151-154.

Franklin, C., P. Vaillancourt, M. K. Yau, and P. Bartello, 2005: Collision rates of cloud droplets in turbulent flow. J. Atmos. Sci., 62, 2451-2466.

Girimaji, S. S., and S. B. Pope, 1990: Material-element deformation in isotropic turbulence. J. Fluid Mech., 220, 427-458.

Grabowski, W., and P. Vaillancourt, 1999: Comments on "Preferential concentration of cloud droplets by turbulence." $J$. Atmos. Sci., 56, 1433-1436.

Grits, B., M. Pinsky, and A. Khain, 2006: Investigation of smallscale droplet concentration inhomogeneities in a turbulent flow. Meteor. Atmos. Phys., 92, 191-204.

Jaczewski, A., and S. P. Malinowski, 2005: Spatial distribution of cloud droplets in a turbulent cloud-chamber flow. Quart. J. Roy. Meteor. Soc., 131, 2047-2062.

Jonas, P., 1996: Turbulence and cloud microphysics. Atmos. Res., 40, 283-306.

Kostinski, A., and R. Shaw, 2001: Scale-dependent droplet clustering in turbulent clouds. J. Fluid Mech., 434, 389-398.

Maxey, M. R., 1987: The gravitational settling of aerosol particles in homogeneous turbulence and random flow field. J. Fluid Mech., 174, 441-465.

McFarquhar, G. M., 2004: The effect of raindrop clustering on collision-induced breakup of raindrops. Quart. J. Roy. Meteor. Soc., 130, 2169-2190.

Pumir, A., 1994: A numerical study of pressure fluctuations in incompressible, homogeneous, isotropic turbulence. Phys. Fluids, 6, 2071-2083.

Reade, W., and L. Collins, 2000: Effect of preferential concentration on turbulent collision rates. Phys. Fluids, 12, 2530-2540.

Saffman, P., and J. Turner, 1956: On the collision of drops in turbulent clouds. J. Fluid Mech., 1, 16-30.

Shaw, R., 2003: Particle turbulence interaction in atmospheric clouds. Annu. Rev. Fluid Mech., 35, 183-227.

Squires, K., and J. Eaton, 1991: Measurements of particle dispersion from direct numerical simulations of isotropic turbulence. J. Fluid Mech., 226, 1-35.

Sundaram, S., and L. Collins, 1997: Collision statistics in an isotropic particle-laden turbulent suspension. J. Fluid Mech., 335, 75-109.

Vaillancourt, P. A., and M. K. Yau, 2000: Review of particleturbulence interactions and consequences for cloud physics. Bull. Amer. Meteor. Soc., 81, 285-298.

Wang, L. P., and M. Maxey, 1993: Settling velocity and concentration distribution of heavy particles in homogeneous isotropic turbulence. J. Fluid Mech., 256, 27-68.

—, A. Wexler, and Y. Zhou, 1998: Statistical mechanical descriptions of turbulent coagulation. Phys. Fluids, 10, 2647-2651.

—, A. Orlando, S. E. Kasprzak, and W. W. Grabowski, 2005: Theoretical formulation of collision rate and collision efficiency of hydrodynamically interacting cloud droplets in turbulent atmosphere. J. Atmos. Sci., 62, 2433-2450.

_ C. N. Franklin, O. Ayala, and W. W. Grabowski, 2006: Probability distributions of angle of approach and relative velocity for colliding droplets in a turbulent flow. J. Atmos. Sci., 63, 881-900.

Wilkinson, M., and B. Mehlig, 2003: Path coalescence transition and its applications. Phys. Rev. E, 68, 1-4.

, and - 2005: Caustics in turbulent aerosols. Europhys. Lett., 71, 186-192.

,$- \ldots$, and V. Bezuglyy, 2006: Caustic activation of rain showers. Phys. Rev. Lett., 97, 1-4. 